\title{
A Mini Challenge: Build a Verifiable Filesystem
}

\author{
Rajeev Joshi and Gerard J. Holzmann \\ Laboratory for Reliable Software*, \\ Jet Propulsion Laboratory, \\ California Institute of Technology, \\ Pasadena, CA 91109, USA \\ \{Rajeev.Joshi, Gerard.Holzmann\}@jpl.nasa.gov \\ http://eis.jpl.nasa.gov/lars
}

\begin{abstract}
We propose tackling a "mini challenge" problem: a nontrivial verification effort that can be completed in 2-3 years, and will help establish notational standards, common formats, and libraries of benchmarks that will be essential in order for the verification community to collaborate on meeting Hoare's 15-year verification grand challenge. We believe that a suitable candidate for such a mini challenge is the development of a filesystem that is verifiably reliable and secure. The paper argues why we believe a filesystem is the right candidate for a mini challenge and describes a project in which we are building a small embedded filesystem for use with flash memory.
\end{abstract}

\section{A Mini Challenge}

The verification grand challenge proposed by Hoare [1 sets the stage for the program verification community to embark upon a collaborative effort to build verifiable programs. At a recent workshop in Menlo Park 2, there seemed to be a consensus that a necessary stepping stone to such an effort would be the development of repositories for sharing specifications, models, implementations, and benchmarks so that different tools could be combined and compared.

We believe that the best way of reaching agreement on common formats and forging the necessary collaborations to build such a repository is to embark upon a shorter-term "mini challenge": a nontrivial verification project that can nonetheless be completed in a short time. An ideal candidate for such a mini challenge would have several characteristics: (a) it would be of sufficient complexity that traditional methods such as testing and code reviews are inadequate to establish its correctness, (b) it would be of sufficient simplicity that specification, design and verification could be completed by a dedicated team in a relatively short time, say 2-3 years, and (c) it would be of sufficient importance

\footnotetext{
* The work described in this paper was carried out at the Jet Propulsion Laboratory, California Institute of Technology, under a contract with the National Aeronautics and Space Administration.
} 
that successful completion of the mini challenge would have an impact beyond the verification community.

At the Menlo Park workshop, some participants (notably Amir Pnueli) suggested that a suitable candidate would be the verification of the kerne 1 of the Linux operating system [3. While the task of verifying the Linux kernel undoubtedly meets conditions (a) and (c) above, it does not meet condition (b). In fact, given that the current Linux kernel is well over 4 million lines of source code, it seems a tall order to write a formal specification for it within 2 years, much less verify the correctness of the implementation. Instead, we propose that a more suitable candidate for such a mini challenge would be the development of a verifiable filesystem. We believe there are several reasons why a filesystem is more attractive as a first target for verification than an operating system kernel.

Firstly, most modern filesystems have a clean, well-defined interface, conforming to the POSIX standard [4, which has been in use for many years. Thus writing a formal specification for a POSIX-compliant filesystem would require far less effort than writing a kernel specification. In fact, one could even write an abstract reference filesystem implementation which could be used as the specification for a verification proof based on refinement.

Secondly, since the underlying data structures and algorithms used in filesystem design are very well understood, a verifiable filesystem implementation could conceivably be written from scratch. Alternatively, researchers could choose any of several existing open-source filesystems and attempt to verify them. This makes filesystem verification attractive, since it allows participation by both those researchers interested in a posteriori verification, as well as those interested in "constructing a program and its proof hand-in-hand".

Thirdly, although filesystems comprise only a small portion of an operating system, they are complex enough that ensuring reliability in the presence of concurrent accesses and unexpected power failures is nontrivial. Indeed, recent work by Yang et al shows that many popular filesystems in widespread use have serious bugs that can have devastating consequences, such as deletion of the system root directory 7 .

Finally, since almost all data on modern computers is now managed by filesystems, their correctness is of great importance, both from the standpoint of reliability as well as security. Development of a verified filesystem would therefore be of great value even beyond the verification community.

\section{Directions and Challenges}

The goal of the proposed mini challenge is to build a verifiable filesystem. In particular, we are interested in the problem of how to write a filesystem whose correctness can be checked using automated verification tools. After decades of experience with automatic program verification, we know that such an effort inevitably requires that key design knowledge be captured and expressed in machine readable forms that can be used to guide the verification tools. This

\footnotetext{
${ }^{1}$ Actually, Pnueli suggested verifying "Linux"; we assume he meant the Linux kernel.
} 
includes (a) a formal behavioral specification of the functionality provided by the filesystem, (b) a formal elaboration of the assumptions made of the underlying hardware, and (c) a set of invariants, assertions and properties concerning key data structures and algorithms in the implementation. We discuss each of these artifacts below.

Specification. Most modern filesystems are written to comply with the POSIX standard [4] for filesystems. This standard specifies a set of function signatures (such as creat, open, read, write), along with a behavioral description of each function. However, these behavioral descriptions are given as informal English prose, and are therefore too ambiguous and incomplete to be useful in a verification effort. The first task therefore is to write a formal specification of the POSIX standard (or at least of a substantial portion of the standard) either as a set of logical properties or as an abstract reference implementation. Such formal specifications have been written in the past: for instance, by Morgan and Sufrin [5], who wrote a specification of the UNIX filesystem in Z, and by Bevier, Cohen and Turner [6], who wrote a specification for the Synergy filesystem in Z (and also partially in ACL2). Although these specifications did not completely model POSIX behavior (for instance, neither completely modeled error codes, nor file permissions), they could serve as starting points for developing a more complete specification.

Assumptions about underlying hardware. In order to provide a rigorous formal statement of the properties of the filesystem (especially its robustness with respect to power failure), it is necessary to rely on certain behavioral assumptions about the underlying hardware. In order to make the filesystem useful, it is necessary to understand what assumptions can reasonably be made about typical hardware such as hard drives or flash memory. These assumptions need to be explicitly identified and clearly stated, as opposed to used implicitly in correctness proofs (as is often the case). In the ideal situation, the filesystem would be usable with different types of hardware, perhaps providing different reliability guarantees.

Properties of data structures and procedures. As noted before, an attractive feature of the proposed mini challenge is that one could either write a verifiable filesystem from scratch, or verify an available filesystem. In either case, however, in order to use automatic checking tools to prove nontrivial correctness properties of the implementation, it will inevitably be necessary to identify and express design properties such as data structure invariants, annotations describing which locks protect which data, and pre- and post-conditions for library functions. Most typical filesystems require use of many common data structures such as hash tables, linked lists and search trees. A proof of filesystem correctness would therefore result in development of libraries of formally stated properties and proofs about these data structures, which would be useful in other verification efforts as well. 


\section{A Reliable Flash Filesystem for Flight Software}

At the NASA/JPL Laboratory for Reliable Software (LaRS), we are interested in the problem of building reliable software that is less reliant on following traditional ad-hoc processes and more reliant on use of automated verification tools. As part of this effort, we are currently engaged in a pilot project to help build a reliable filesystem for flash memory, for use as nonvolatile storage on board future missions.

Flash memory has recently become a popular choice for use on spacecraft as nonvolatile storage for engineering and data products, since it has no moving parts, consumes low power and is easily available. There are two common types of flash memory, NAND flash and NOR flash 8 . While NOR flash is more reliable and easier to program, it has lower density and poor write and erase times, and is therefore less attractive as a data storage device. While it is possible to design flight software to use flash memory directly as a raw device, it is typically much easier to write robust flight software on top of a filesystem layer that provides common file operations for creating, reading and writing files and directories. In fact, the flight software on several recent NASA missions, such as the Mars Exploration Rovers and Deep Impact, uses a filesystem to access flash memory.

Building a robust flash filesystem, however, is a nontrivial task. Performance dictates the use of caches and write buffers, which increase the danger of inconsistencies in the presence of concurrent thread accesses and unexpected power failures. To add to the challenge, flash memory, especially NAND flash memory, requires certain additional issues to be addressed such as arbitrary bit flips, blocks that unexpectedly become "bad" (i.e., permanently unusable), and limited lifetimes (block usually become bad after they have been erased a certain number of times, typically 100,000). In addition, a flash filesystem written for use on a spacecraft must obey additional constraints; for instance, flight software is typically allowed to allocate memory only during initialization.

The goal of our pilot project is to build a robust flash filesystem by following a design methodology that is based on documenting as much as possible in a machine readable form that is amenable to automatic verification. Thus the intent is not only to build a working filesystem, but also to produce key design documents in machine-readable forms that can be used by automated verification tools. Although less ambitious than the mini challenge we have described above (which is aimed at building a general purpose filesystem), our project has similar interests and goals with the mini challenge we have proposed.

\section{Summary}

An important first step toward the Verification Grand Challenge is the development of a repository containing specifications, models and implementations. We believe the best way to develop this repository is to tackle a "mini challenge" that can be completed in a short period of time, around 2-3 years. An excellent 
candidate for such a mini challenge seems to be the development of a verifiable filesystem that is both reliable and secure. Since filesystems are well-defined and well-understood, different research teams can take different approaches to building such a verifiable filesystem, from building it from scratch to verifying one of many available filesystems. We believe that the problem is well-suited as a mini challenge for the verification community and will serve as a starting point for the grand verification challenge.

\section{References}

1. Hoare, T.: The Verifying Compiler: A Grand Challenge for Computing Research. Journal of the ACM 50(1), 63-69 (2003)

2. Workshop on the Verification Grand Challenge, SRI International, Menlo Park, CA (February 2005), http://www.csl.sri.com/users/shankar/VGC05

3. Pnueli, A.: Looking Ahead, Presentation at the Workshop on The Verification Grand Challenge, SRI International, Menlo Park, CA (February 2005), http://www.csl.sri.com/users/shankar/VGC05/pnueli.pdf

4. The Open Group, The POSIX 1003.1, 2003 Edition Specification, http://www.opengroup.org/certification/idx/posix.html

5. Morgan, C., Sufrin, B.: Specification of the UNIX Filing System. IEEE Transactions on Software Engineering SE-10(2), 128-142 (1984)

6. Bevier, W.R., Cohen, R., Turner, J.: A Specification for the Synergy File System, Technical Report 120, Computational Logic, Inc., (September 1995)

7. Yang, J., Twohey, P., Engler, D., Musuvathi, M.: Using Model Checking to Find Serious File System Errors. In: Proceedings of the Conference on Operating Systems Design and Implementation (OSDI), San Francisco, pp. 273-288 (December 2004)

8. Data I/O, A Collection of NAND Flash Application Notes, Whitepapers and Articles, http://www.data-io.com/NAND/NANDApplicationNotes.asp

\section{A Discussion on Rajeev Joshi's Presentation}

\section{Jayadev Misra}

[Inaudible question]

\section{Rajeev Joshi}

I believe, the specs could be written by a group of five people-any five people in this room-in less than six months. So, how long would it take to write a file system? I think the big problem is getting the machine-verifiability part of it. So, if we go back and think about the level of ambition, I think the first one should be doable by a group of less than ten people in a year. But remember, we are not solving the general problem; we are solving the problem in the context of a filesystem. So, the harder part is as you go down the list. I do not really have an answer. I would be guessing, and I do not know what to guess. 


\section{Greg Nelson}

Rajeev, when you talked about the options for translating the POSIX English language spec into a formal form, you mentioned two options, neither of which seems to me to be the translation into preconditions and postconditions and modifiers, because this would have been the first thing I would think of. Can you educate me or tell me, how I suffer this disconnect?

\section{Rajeev Joshi}

That is because I should have said: "like pre- and postconditions or temporal logical properties or a reference implementation".

\section{Tevfik Bultan}

You mentioned, that there was a paper that found errors in the existing filesystems. So, what types of errors, you listed a categorization of types of correctness, could we check? So, did they find null dereferences or...

\section{Rajeev Joshi (interrupts)}

No, it is more complex. They used a kind of model checking and they found issues where you can have kernel panics, or you could have data corruption, metadata corruption, which should cause losing the system root directory under certain conditions with multiple threads running. So, no, they used software model checking, essentially.

\section{Peter O'Hearn}

Isn't this awfully ambitious as a first step? The verification should be automatic, I take it.

\section{Rajeev Joshi}

I don't think that in the end we will have a tool that will work on an arbitrary computer program, but we will have something, that will essentially be tailored for this filesystem. I think part of the problem is doing it in a way that all the machine-readable artifacts are published somewhere, which is the issue of setting up a repository. So, somebody who writes the specification has to write it in a format, so that other people know what the format is and have agreed upon it.

\section{Peter O'Hearn}

So, you are proposing not to test the proof tools so much as the specifications. 


\section{Rajeev Joshi}

Well, again it depends on your level of ambition when you say, "proof tools". I don't know how far we can go. If we stop with model checking then, if you write a set of invariants and you say, "well, we model-checked and we guarantee that the model checker has actually covered the entire space", then that is some level of verification I think we could reach. I don't think it is too ambitious from that point of view. I think the harder problem is to make people agree on formats. I mean, that's a fact of life. I have seen this before in other instances that it is not always... [sentence incomplete]

\section{Peter O'Hearn (interrupts)}

Now, if you ask for automatic verification, then the difficulty is very great, but if you would allow manual verification, then I would agree with you. You could do it with some number of man-years. But if the project is to have nearly automatic methods, then your proposal seems like an extremely difficult one as a first step.

\section{Rajeev Joshi}

Ok... So, we can check it manually, maybe.

\section{Egon Börger}

Since you are speaking about details: I was surprised that you seem to separate the core of specification from what you mention under "design", namely, concurrency, fault tolerance, asynchrony. I think they should be part of the specification, because of the many things you will have to handle at that level. This is something where you have to connect to the operating system somehow, or your scheduling mechanism. And this may break your spec. So, it should be part of the spec, I guess.

\section{Rajeev Joshi}

Yes that is of course one of the hard problems in computing science in verification: What assumptions can you make? Under what assumptions is your program going to work? And I think that's hard. Yes, it's true-since the filesystem typically runs as part of the operating system-that how the operating system manages threads, for instance, is an issue. What guarantees the operating system provides against threads writing on each other's data would be an issue. Is that the kind of thing you're asking about?

\section{Egon Börger}

Yes, that is exactly the kind of reason why I believe it should be part of your spec. You should analyze this really mathematically, because if you look at what happened to Java, with thread handling in Java, the same you have in C\#. When you go to the thread handling mechanism, it is poorly described, and as a 
programmer, you are left with almost no knowledge of what is going on, except you know all the details of compilers and maybe operating systems. The same thing would happen to your filesystem spec. If you separate specification from what has to do with asynchronous communication or whatever, then you can throw away your spec later on. It does not tell you the real story. It is just a purely functional view under the assumption that all the accesses are safe and secure, or whatever.

\section{Rajeev Joshi}

Yes, I think that is an inherent property of specifications. And I think one just writes with a certain model in mind.

\section{Egon Börger}

So, you need a communication model and an action model...

\section{Rajeev Joshi}

I don't think that is necessary. But for instance, as Greg mentioned, if you write pre- and postconditions, then it's pretty well defined what the open()-operation does with the pre- and postconditions. So, you can just check that the code for the open() will satisfy the pre- and postconditions. Now, of course, if you are running something concurrently with an open(), say if you are running a create(), then those guarantees don't hold, but that is a different problem. So, I think it depends on, again, your level of ambition. You can say that we have checked it so that it is correct with respect to pre- and postcondition semantics, but this does not mean, it is correct with respect to everything else.

\section{Egon Börger}

The only thing I wanted to point out is that I would not relegate it to just design; I would do this as high up as possible. 